Homology, Homotopy and Applications, vol.6(1), 2004, pp.237-267

\title{
THE GEOMETRY OF CONFIGURATION SPACES FOR CLOSED CHAINS IN TWO AND THREE DIMENSIONS
}

\author{
R. JAMES MILGRAM AND J. C. TRINKLE \\ (communicated by Gunnar Carlsson)
}

\begin{abstract}
In this note we analyze the topology of the spaces of configurations in the euclidian space $\mathbb{R}^{n}$ of all linearly immersed polygonal circles with either fixed lengths for the sides or one side allowed to vary. Specifically, this means that the allowed maps of a $k$-gon $\left\langle l_{1}, l_{2}, \ldots, l_{k}\right\rangle$ where the $l_{i}$ are the lengths of the successive sides, are specified by an ordered $k$ tuple of points in $\mathbb{R}^{n}, P_{1}, P_{2}, \ldots, P_{k}$ with $d\left(P_{i}, P_{i+1}\right)=l_{i}$, $1 \leqslant i \leqslant k-1$ and $d\left(P_{k}, P_{1}\right)=l_{k}$. The most useful cases are when $n=2$ or 3 , but there is no added complexity in doing the general case. In all dimensions, we show that the configuration spaces are manifolds built out of unions of specific products $\left(S^{n-1}\right)^{H} \times I^{(n-1)(k-2-H)}$, over (specific) common sub-manifolds of the same form or the boundaries of such manifolds. Once the topology is specified, it is indicated how to apply these results to motion planning problems in $\mathbb{R}^{2}$.
\end{abstract}

\section{Introduction}

Polygonal circles in $\mathbb{R}^{2}$ or $\mathbb{R}^{3}$ with $k$-edges are called $k$-bar mechanisms in mechanical engineering, and they often arise with one of the edges fixed. In the latter case they are called closed $(k-1)$-chains. Generally the lengths of the edges are assumed to be fixed, but if the length of one of the links is allowed to vary between $l_{i}$ and $l_{i}^{\prime}$, then this link is called a prismatic joint. The space of configurations, particularly in the case of closed chains, is very important in areas like robotics where motions of these mechanisms from an initial position to a final position often with special constraints like avoiding certain points or some self-intersections - are objects of essential interest. Such motions are best regarded as paths in the configuration space or suitable subspaces. We will describe these connections and related problems in $\S 2$.

First author partially supported by Sandia National Laboratories and the NSF.

Second author supported by Sandia National Laboratories.

Received August 26, 2003, revised May 31, 2004; published on June 21, 2004.

2000 Mathematics Subject Classification: 55R80.

Key words and phrases: Configuration spaces, linkages.

(C) 2004, R. James Milgram and J. C. Trinkle. Permission to copy for private use granted. 


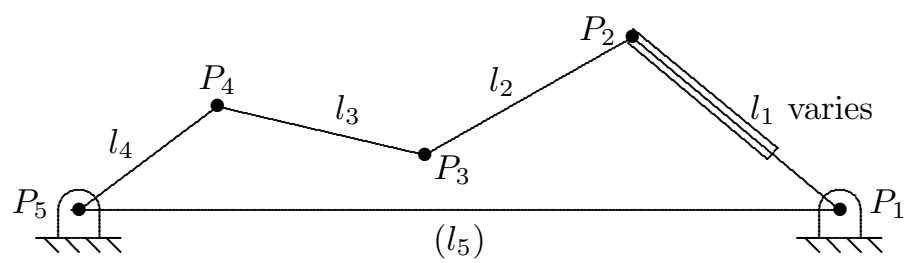

Figure 1: Five bar mechanism with one prismatic joint

Equivalent forms of the configuration space and key subspaces: Let $\mathcal{B}\left(l_{1}, \ldots, l_{k}\right)$ denote the space of all configurations of closed chains with $k$ links in $\mathbb{R}^{n}$, the first link of length $l_{1}$, the second of length $l_{2}$, etc. As described in the abstract, each such configuration is given by an ordered $k$-tuple of points in $\mathbb{R}^{n}$, $P_{1}, \ldots, P_{k}$, where the vector $P_{2}-P_{1}$ has length $l_{1}, P_{3}-P_{2}$ has length $l_{2}$, etc., and $l_{k-1}$ and $P_{1}-P_{k}$ has length $l_{k}$. These vectors are completely determined by giving their lengths and the unit vectors $\vec{e}_{1}, \ldots, \vec{e}_{k}$ in their respective directions. Conversely, a $k$-tuple of unit vectors $\left(\vec{e}_{1}, \ldots, \vec{e}_{k}\right) \in\left(S^{n-1}\right)^{k}$ and an initial point $P_{1} \in \mathbb{R}^{n}$ determines an element of $\mathcal{B}\left(l_{1}, \ldots, l_{k}\right)$ if and only if the vector sum

$$
\sum_{1}^{k} l_{j} \vec{e}_{j}=\overrightarrow{0}
$$

Let $\mathcal{D}\left(l_{1}, \ldots, l_{k}\right) \subsetneq\left(S^{n-1}\right)^{k}$ be the set of $k$-tuples $\left(\vec{e}_{1}, \ldots, \vec{e}_{k}\right)$ with $\sum_{1}^{k} l_{j} \vec{e}_{j}=$ $\overrightarrow{0}$. Then the remarks above show that there is a homeomorphism between $\mathbb{R}^{n} \times$ $\mathcal{D}\left(l_{1}, \ldots, l_{k}\right)$ and $\mathcal{B}\left(l_{1}, \ldots, l_{k}\right)$. Consequently, since the exchange map, exchanging the $i^{\text {th }}$ and $j^{\text {th }}$ coordinates in $\mathcal{D}\left(l_{1}, \ldots, l_{k}\right)$, identifies

$$
\mathcal{D}\left(l_{1}, \ldots, l_{i}, \ldots, l_{j}, \ldots, l_{k}\right) \text { with } \mathcal{D}\left(l_{1}, \ldots, l_{j}, \ldots, l_{i}, \ldots, l_{k}\right),
$$

the order of the links does not matter if we know the initial point $P_{1}$. On the other hand, if we map the elements of $\mathcal{B}\left(l_{1}, \ldots, l_{k}\right)$ to $\mathbb{R}^{n}$ by taking each configuration to its initial point, $P_{1}$, this is a (trivial) fibration with fiber the set of configurations where $P_{1}$ is fixed, $\mathcal{B}\left(l_{1}, \ldots, l_{k}, P_{1}\right)$. Thus, the initial point does not matter either, and

$$
\mathcal{B}\left(l_{\sigma(1)}, \ldots, l_{\sigma(k)}, P\right) \text { is homeomorphic to } \mathcal{B}\left(l_{1}, \ldots, l_{k}, Q\right)
$$

for any permutation $\sigma$ of $(1, \ldots, k)$ and any two points $P, Q$ in $\mathbb{R}^{n}$.

The action of the Euclidian group on $\mathcal{B}\left(l_{1}, \ldots, l_{k}\right)$ : The oriented Euclidean group of rigid motions, $S E_{n}=\mathbb{R}^{n}: S O_{n}$, (where $H: G$ is the semi-direct product and the action of $S O_{n}$ on $\mathbb{R}^{n}$ is the usual one) acts on the configuration space $\mathcal{B}\left(l_{1}, \ldots, l_{k}\right)$ by

$$
g\left(P_{1}, \ldots, P_{k}\right)=\left(g\left(P_{1}\right), \ldots, g\left(P_{k}\right)\right) .
$$

Writing $\mathcal{B}\left(l_{1}, \ldots, l_{n}\right)=\mathbb{R}^{n} \times \mathcal{B}\left(l_{1}, \ldots, l_{k}, \overrightarrow{0}\right)$, and $g$ as the composition of a translation $T$ and an element $h \in S O_{n}$, we have that this action can be written as

$$
\left.\left(P,\left(\vec{e}_{1}, \ldots, \vec{e}_{k}\right)\right) \mapsto\left(T(P), h\left(\vec{e}_{1}\right), \ldots, h\left(\vec{e}_{k}\right)\right)\right)
$$


and the moduli space of these immersed polygonal circles is defined to be the quotient of this action. In all dimensions it is the same as the quotient of $\mathcal{B}\left(l_{1}, \ldots, l_{k}, \overrightarrow{0}\right)$ by $\mathrm{SO}_{n}$.

The relationship between closed $(k-1)$-chains and $\mathcal{B}\left(l_{1}, \ldots, l_{k}\right): \mathcal{B}\left(l_{1}, \ldots, l_{k}, \overrightarrow{0}\right)$ projects to $S^{n-1}$ by taking $\left(\vec{e}_{1}, \ldots, \vec{e}_{k}\right)$ to $\vec{e}_{k}$. This is a fibration with fiber the set of configurations where $\vec{e}_{k}$ is fixed, the configuration space of closed $(k-1)$-chains,

$$
\mathcal{C}\left(l_{1}, \ldots, l_{k-1} \mid l_{k} \vec{e}_{k}\right) .
$$

The action of $S O_{n}$ on $\mathcal{B}\left(l_{1}, \ldots, l_{k}\right)$ restricts to give an action of the subgroup $S O_{n-1}$ that fixes $\vec{e}_{k}$ on $\mathcal{C}\left(l_{1}, \ldots, l_{k-1} \mid l_{k} \vec{e}_{k}\right)$. Using this action, the fibration above,

$$
\mathcal{C}\left(l_{1}, \ldots, l_{k-1} \mid l_{k} \vec{e}_{k}\right) \longrightarrow \mathcal{B}\left(l_{1}, \ldots, l_{k}, \overrightarrow{0}\right) \longrightarrow S^{n-1},
$$

is the associated fibration to the usual bundle

$$
S O_{n-1} \longrightarrow S O_{n} \stackrel{\pi}{\longrightarrow} S^{n-1}
$$

where $\pi(g)=g\left(\vec{e}_{x}\right)$, and $\vec{e}_{x}$ is the unit vector in the $x$-direction. Consequently, we can write the fibration in the form

$$
\mathcal{C}\left(l_{1}, \ldots, l_{k-1} \mid l_{k} \vec{e}_{x}\right) \longrightarrow \mathcal{C}\left(l_{1}, \ldots, l_{k-1} \mid l_{k} \vec{e}_{x}\right) \times_{S O_{n-1}} S O_{n} \longrightarrow S^{n-1}
$$

The fibration is trivial when $n=2$ since $S O_{1}=\{1\}$, but is non-trivial for $n \geqslant 3$. Note also that for $n>3$ the space $\mathcal{C}\left(l_{1}, \ldots, l_{k-1} \mid l_{k} \vec{e}_{x}\right)$ is never free under the action of $S O_{n-1}$ since it will contain configurations that lie in an $\mathbb{R}^{n-2}$ containing $\vec{e}_{x}$, which will be fixed under a copy of $1 \times S_{2} \subset S O_{n-3} \times S O_{2}$ where $S_{n-3}$ fixes the pair $\left(\mathbb{R}^{n-2}, \vec{e}_{x}\right)$ and the $S_{2}$ acts on the orthogonal $\mathbb{R}^{2}$.

Also, note that the quotient of $\mathcal{B}\left(l_{1}, \ldots, l_{k}\right)$ by the Euclidian group is the quotient of $\mathcal{C}\left(l_{1}, \ldots, l_{k-1} \mid l_{k} \vec{e}_{k}\right)$ by $S O_{n-1}$, but only in the case where $n=2$ can we identify $\mathcal{C}\left(l_{1}, \ldots, l_{k-1} \mid l_{k} \vec{e}_{k}\right)$ with this quotient.

The configuration space of a closed chain: In this paper our focus is on the spaces $\mathcal{C}\left(l_{1}, \ldots, l_{k-1} \mid l_{k} \vec{e}_{x}\right)$. Note that we can describe $\mathcal{C}\left(l_{1}, \ldots, l_{k-1} \mid l_{k} \vec{e}_{x}\right)$ as the subspace of $\left(S^{n-1}\right)^{k-1}$ consisting of vectors $\left(\vec{e}_{1}, \ldots, \vec{e}_{k-1}\right)$ with

$$
\sum_{1}^{k-1} l_{i} \vec{e}_{i}=-l_{k} \vec{e}_{x}
$$

Consequently the order of $l_{1}, \ldots, l_{k-1}$ does not matter in $\mathcal{C}\left(l_{1}, \ldots, l_{k-1} \mid l_{k} \vec{e}_{x}\right)$. For $n \geqslant 3$ we are not able to give a natural identification of the space

$$
\mathcal{C}\left(l_{1}, \ldots, l_{k-l} \mid l_{k} \vec{e}_{x}\right) \text { with } \mathcal{C}\left(l_{1}, \ldots, l_{k-2}, l_{k} \mid l_{k-1} \vec{e}_{x}\right),
$$

but the triviality of the fibration in the previous paragraph when $n=2$ implies that there is a unique element of the Euclidian group $S E_{2}$ that moves the $i^{\text {th }}$ link so that its final point is $\overrightarrow{0}$ and so that its initial point is on the negative $x$-axis. It follows that order is entirely immaterial for the configuration space of closed chains when $n=2$.

Remark 1.2. In the case where all the lengths are fixed, if we rescale by multiplying all the lengths by the same non-zero constant $\lambda$ the configuration spaces and moduli spaces are again homeomorphic. Consequently, we can assume that $\sum_{1}^{k} l_{i}=1$, all 
the $l_{i}>0$, and if we wish, that the lengths are given in increasing order except possibly for the last link when $n \geqslant 3$. Unless otherwise stated the convention that $\sum l_{i}=1$ will be in force for the remainder of this note whenever we discuss the situation where all the lengths are fixed.

Definition 1.3. Assume that the $l_{i}$ are normalized as above. Then we say that the subset $V=\left(l_{i_{1}}, l_{i_{2}}, \ldots, l_{i_{r}}\right)$ consists of long links if and only if the sum of any two lengths in $V$ is greater than $\frac{1}{2}$.

The following lemma appears in [KM1] and shows that no real $k$-bar can have only one long link:

Lemma 1.4. The ordered sequence $\left\langle l_{1}, l_{2}, \ldots, l_{k}\right\rangle$ with $\sum_{1}^{k} l_{i}=1$ and $l_{i}>0$ for all $i$, has a non-empty configuration space in $\mathbb{R}^{n}, n \geqslant 2$, if and only if each $l_{i} \leqslant \frac{1}{2}$.

(The proof is elementary. The result is verified for $k=3$ and then the proof for $k>3$ is a direct induction when one observes that for $k>3$, there must be two lengths $l_{i}, l_{j}$ with $l_{i}+l_{j}<\frac{1}{2}$.)

Remark 1.5. It is also possible for the mechanism to have three long links $l_{i}, l_{j}, l_{k}$ so that the sum of any two is $\geqslant \frac{1}{2}$, though it is not possible to have four long links. In the case of three long links we have the important result, [KM1]:

Theorem 1.6. For configurations of a $k$-bar mechanism with fixed lengths in $\mathbb{R}^{2}$ the configuration space is connected if and only if the mechanism does not have three long links. Moreover in the case where the mechanism does have three long links, then the configuration space has exactly two components and each component is a torus $\left(S^{1}\right)^{k-3}$. (For $\mathbb{R}^{n}, n \geqslant 3$, the moduli space is always connected.)

$\mathcal{C}\left(l_{1}, \ldots, l_{k-1} \mid l_{k} \vec{e}_{x}\right)$ is generically a closed manifold, but much more is true.

Theorem 1.7. Suppose given a closed $(k-1)$-chain with fixed lengths $l_{1}, l_{2}, \ldots, l_{k-1}$ and base length $l_{k}$ which is allowed to vary.

(a) Except for a finite number of choices for $l_{k}$ with $l_{k}<\sum_{1}^{k-1} l_{j}, \mathcal{C}\left(l_{1}, \ldots, l_{k-1}\right.$ $\left.\mid l_{k} \vec{e}_{k}\right)$ is a closed compact manifold of dimension $(n-1)(k-2)-1$, and is connected for $n \geqslant 3$.

(b) Whenever $\mathcal{C}\left(l_{1}, \ldots, l_{k-1} \mid l_{k} \vec{e}_{k}\right) \subset\left(S^{n-1}\right)^{k}$ is a manifold, it is the boundary of a manifold $W^{(n-1)(k-2)} \subset\left(S^{n-1}\right)^{k}$ which is given as a finite union of submanifolds of the form

$$
\left(S^{n-1}\right)^{s} \times I^{(n-1)(k-s-2)},
$$

each of which is taut in the sense that its integral homology injects to a direct summand of $H_{*}(W ; \mathbb{Z})$ and the sum of all the images is exactly $H_{*}(W ; \mathbb{Z})$. (The set of $s$ that occur depend on the lengths in a fairly direct way and is described in Theorem 1.12.) 


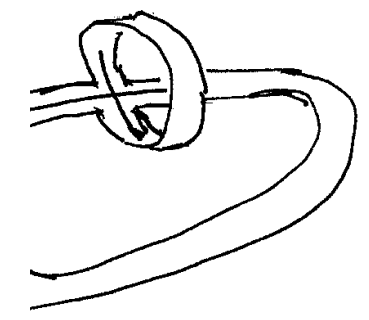

Figure 2: The (coordinate) union of two copies of $S^{1} \times I$

This union is constructed as follows. Whenever two such pieces, $\left(S^{n-1}\right)^{s_{1}} \times$ $I^{(n-1)\left(k-s_{1}-2\right)}$ and $\left(S^{n-1}\right)^{s_{2}} \times I^{(n-1)\left(k-s_{2}-2\right)}$, intersect, their intersection is a common (coordinate) sub-manifold of the form

$$
\left(S^{n-1}\right)^{l} \times I^{\left.(n-1)\left[\left(s_{1}-l\right)+\left(s_{2}-l\right)+k-s_{1}-s_{2}+l-2\right)\right]}=\left(S^{n-1}\right)^{l} \times I^{(n-1)[k-l-2]}
$$

with trivial normal bundle and, as indicated in 1.8, a certain number of the coordinates in the normal bundle point in the direction of the first piece, while a complementary set point in the direction of the second. Also, any two of these sub-manifolds have at least one point in common, $\left(S^{n-1}\right)^{0}$.

Here, coordinate sub-torus simply means that we fix a finite number of the product coordinates in $\left(S^{n-1}\right)^{k-1}$ and allow the remaining points to vary over all possible values. Also, the structure of this finite union of sub-tori (shorthand for products of spheres) is entirely explicit, consisting of a finite number of maximal sub-tori together with all their possible intersections, and the set of maximal sub-tori is given as a combinatorial function of the lengths. The precise descriptions are given in Theorem 1.12, and the proof follows directly from 1.12. In turn, the proof of 1.12 will require all of sections 5 - 9, with introductory material given in sections $3-4$.

Remark 1.9. In specific cases, it is quite direct to determine the exact structure of these $W^{m}$.

Example 1.10. We give some examples for $\mathbb{R}^{2}$.

(a) If all the $l_{i}, i<k$, are equal, then the $W^{k-2}$ are thickenings of the full $s$ skeleton of $\left(S^{1}\right)^{(k-1)}$ for $s \leqslant[(k-1) / 2]$. Compare [K1], [KTT], [KT], which use very different techniques. (A thickening of $X$ is a manifold $M$ containing $X$ together with a deformation retraction of $M$ to $X$.)

(b) If there are three long edges, $W$ has the form

$$
\left(S^{1}\right)^{k-3} \times I \text {. }
$$

Moreover, from the description above, the intersection pairing is easily described in any specific case. Applying the homology long exact sequence of the pair

$$
\left(W, \mathcal{C}\left(l_{1}, \ldots, l_{k-1} \mid l_{k} \vec{e}_{x}\right)\right)
$$


and Poincaré duality, the intersection pairing determines $H_{*}\left(\mathcal{C}\left(l_{1}, \ldots, l_{k-1} \mid l_{k} \vec{e}_{x}\right), \mathbb{F}\right)$, so the homology structure of the configuration space of any closed chain can be regarded as completely understood.

We conclude this paragraph with a few remarks about the $S O_{n-1}$ action on the configuration space $\mathcal{C}\left(l_{1}, \ldots, l_{k-1} \mid l_{k} \vec{e}_{x}\right)$. A number of authors have shown that a necessary and sufficient condition that $S O_{2}$ act freely is that $\mathcal{C}\left(l_{1}, \ldots, l_{k-1} \mid l_{k} \vec{e}_{x}\right)$ be a manifold when $n=3$. Moreover, the configuration space fibers over the moduli space with fiber $S^{1}$, so the configuration space is homotopy equivalent to the complement of the 0 -section in a complex line bundle over the moduli space. In this connection we note the following intriguing fact: in [KM2] it is shown that in the case where the action of $S O(2)$ is free, the quotient manifold has a complex structure. It is natural to wonder if the line bundle above is actually holomorphic, and what its interpretation with respect to this complex structure might be.

The configurations of closed chains with one prismatic joint: The key to our analysis in 1.7 involves the analysis of the configuration spaces where the base link $l_{k} \vec{e}_{x}$ is allowed to vary in size $0<a \leqslant l_{k} \leqslant b$. We denote these spaces

$$
\mathcal{C}\left(l_{1}, \ldots, l_{k-1} \mid m \vec{e}_{x}, a \leqslant m \leqslant b\right) .
$$

When $l_{1}, \ldots, l_{k-1}$ are fixed,

$$
\mathcal{C}\left(l_{1}, \ldots, l_{k-1} \mid m \vec{e}_{x}, a \leqslant m \leqslant b\right)
$$

is a manifold with boundary the disjoint union

$$
\mathcal{C}\left(l_{1}, \ldots, l_{k-1} \mid a \vec{e}_{x}\right) \sqcup \mathcal{C}\left(l_{1}, \ldots, l_{k-1} \mid b \vec{e}_{x}\right)
$$

except for a finite number of possible values for $a$ and $b$ with $0<a<b<\sum_{1}^{k-1} l_{j}$. (The exact values are given in 1.12(a).)

There is only one configuration with $l_{k}=\sum_{1}^{k-1} l_{j}$, the one where each $\vec{e}_{j}=-\vec{e}_{x}$. When $b$ takes this value it turns out that $\mathcal{C}\left(l_{1}, \ldots, l_{k-1} \mid m \vec{e}_{x}, a \leqslant m \leqslant \sum_{1}^{k-1} l_{j}\right)$ is a manifold with boundary $\mathcal{C}\left(l_{1}, \ldots, l_{k-1} \mid a \vec{e}_{x}\right)$ except for the finite number of values for $a$ when $\mathcal{C}\left(l_{1}, \ldots, l_{k-1} \mid a \vec{e}_{x}\right)$ is not a manifold.

A reason for the importance of these configuration spaces is that the manifold

$$
W^{(n-1)(k-2)}
$$

in Theorem 1.7 is identified with

$$
\mathcal{C}\left(l_{1}, \ldots, l_{k-1} \mid m \vec{e}_{x}, l_{k} \leqslant m \leqslant \sum_{1}^{k-1} l_{j}\right) .
$$

In fact, we have

\section{Theorem 1.12.}

(a) $W^{(n-1)(k-2)}$ in 1.11 is a manifold with boundary if and only if there is no subset $\left\{i_{1}, \ldots, i_{r}\right\} \in\{1, \ldots, k\}$ so that $2 \sum_{1}^{r} l_{i_{j}}=\left(\sum_{1}^{k-1} l_{j}\right)-l_{k}$

(b) If $W^{(n-1)(k-2)}$ in 1.11 is a manifold with boundary, then the set of coordinate tori that occur as building blocks for $W$ are in one to one correspondence with the maximal subsets $\left\{i_{1}, \ldots, i_{r}\right\} \subsetneq\{1, \ldots, k-1\}$ so that $2 \sum_{1}^{r} i_{j}<\sum_{1}^{k} l_{j}-l_{k}$. 
(c) For each maximal subset in 1.12(b), the corresponding building block is a product $\left(S^{n-1}\right)^{r} \times I^{(n-1)(k-r-2)} \subset\left(S^{n-1}\right)^{k-1}$, and, in $\left(S^{n-1}\right)^{k}$ is homotopic to the sub-product $S_{i_{1}}^{n-1} \times S_{i_{r}}^{n-1}$ given as the subspace of $\left(S^{n-1}\right)^{k-1}$ where the coordinates not in $\left\{i_{1}, \ldots, i_{r}\right\}$ are equal to $\vec{e}_{x}$.

Example with $k=5$ : We consider a mechanism consisting of 3 links of length 2, 3 , and 4 based at $\overrightarrow{0}$ and a link of length 1 based at $(5,0)$ joined to the endpoint of the first three links. Here is the picture:

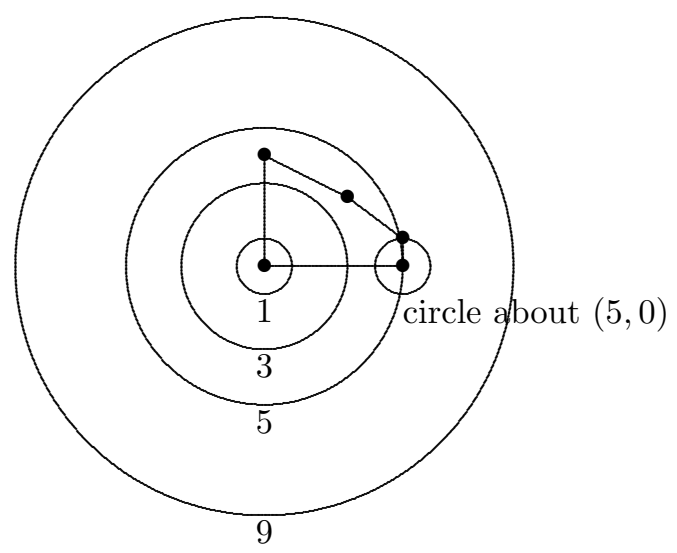

The critical points for the based $(2,3,4)$ mechanism are 9 , a maximum, 5,3 and 1. The little circle of radius 1 centered around $(5,0)$ has two arcs, the upper arc and lower that join at $(6,0)$ and at $(4,0)$. Both go from a distance of 6 from the center to a distance of 4 from the center, and both arcs pass transversally through only one critical circle, the circle of radius $5=4+3-2$. In the outside region, the boundary of the inverse image of each arc is a single circle, and the inside boundary is two circles, with only one critical point between so each inverse image is a copy of a single "pair of pants,"

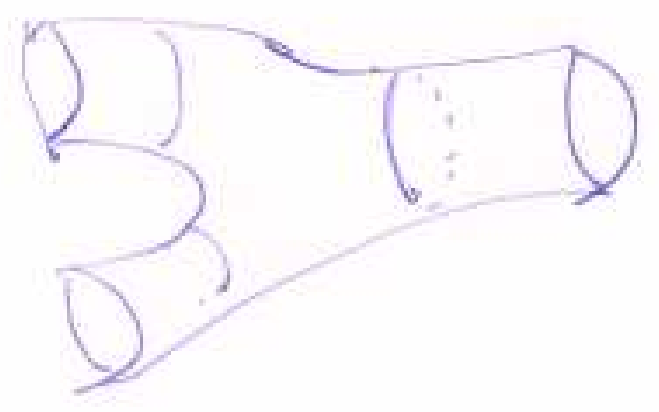


and the entire configuration space is a genus two surface:

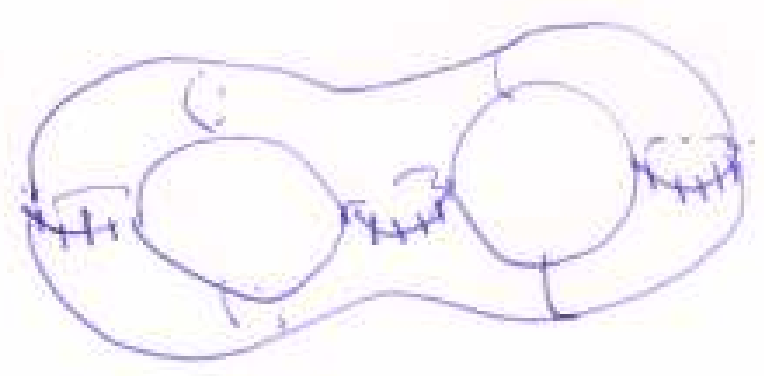

where the dashed lines indicate the points where the three circles on the two copies of the pair of pants are identified.

As an alternative, we look at the mechanism with four links of length 1,2,3,4 and with critical circles of radius 10 maximum, 8,6,4,2 for one negative sign and 4, 2, 0 for 2 negative signs, so 4 has two critical circles above it. Then the path with inverse image the manifold having boundary the configuration space for $(1,2,3,4 \mid 5)$ starts at $(10,0)$ and goes along the $x$-axis to $(5,0)$. Along the way it crosses the critical circles of radius 8 and 6 , both assigned to just one negative link. Hence the result is that the total space of the inverse image of this arc has the homotopy type of the wedge of two circles. Applying Poincare duality, the boundary is a surface with homology that of the two-hole torus, so it is this surface.

In the special case where $n=2$ we will show - as illustrated above - that we can always realize

$$
\mathcal{C}\left(l_{1}, \ldots, l_{k-1} \mid l_{k} \vec{e}_{x}\right), l_{1} \leqslant l_{2} \leqslant \cdots \leqslant l_{k}
$$

as the union of two copies of

$$
\mathcal{C}\left(l_{2}, \ldots, l_{k-1} \mid m \vec{e}_{x}, l_{k}-l_{1} \leqslant m \leqslant \min \left(l_{k}+l_{1}, \sum_{2}^{k-1} l_{j}\right)\right)
$$

over their common boundary.

Theorem 1.14. Let $n=2$. Suppose all the edges have fixed length, suppose that the assumptions of the above theorem are satisfied, and suppose, moreover, that there are two "long edges", $l_{i}$ and $l_{j},(i \neq j)$, so that $l_{i}+l_{j}>\frac{1}{2}$. Then the configuration space for the associated $k$-bar mechanism is the double along the boundary of the thickening given in 1.13.

As an example, when $n=2$ and $k=5$, if there are two long edges, the space $\mathcal{C}\left(l_{1}, \ldots, l_{4} \mid l_{5} \vec{e}_{x}\right)$ is the double over the boundary of one of the manifolds in Figure 3 below. 


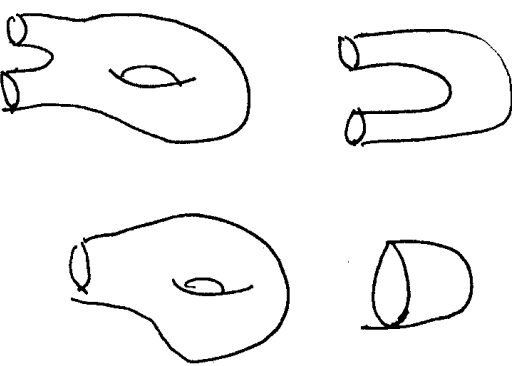

\section{Figure 3: The manifolds $W$ with boundaries $\mathcal{C}$-spaces for 4-bars}

In the remaining cases for $n=2$ and $k=5$ the configuration space is the double over the boundary of one of the spaces in Figure 4.
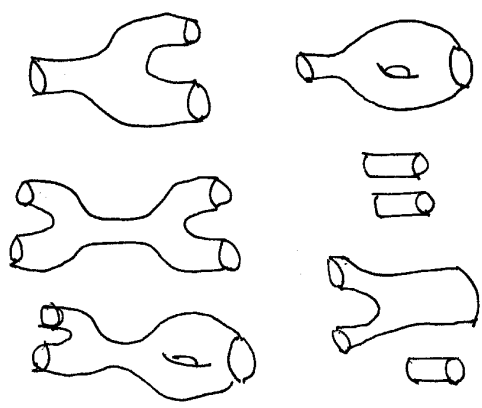

Figure 4:

The proof of 1.12 is based on the careful analysis of the meaning of the critical points of explicit Morse functions constructed on the inverse images of paths in the subspace of $\mathbb{R}^{n}$ which is the image of the end-point map

$$
\left(S^{n-1}\right)^{k-1}, \quad\left(\vec{e}_{1}, \ldots, \vec{e}_{k-1}\right) \mapsto \sum_{1}^{k-1} l_{i} \vec{e}_{i} .
$$

The explicit descriptions of the configuration spaces given above allow for very efficient motion planning in these thickened regions. Specifically, when the topology of the region is sufficiently well understood, it is possible to construct efficient (piecewise geodesic with very few breaks) paths in polynomial time, (roughly $v k^{4}$ ) where $v$ is a constant that depends on the specific configuration space. Details are discussed in section 3 .

The determination of the extremal subsets of $(1, \ldots, k-1)$ associated to the lengths $l_{1}, \ldots, l_{k}$ for these regions can be done in roughly $2^{3 k}$ very direct steps (best possible in general, though when there are very few distinct lengths, the number is much smaller). Of course, this calculation need only be done once for a given mechanism. However, once one knows the configuration space in this detail, more 
efficient paths can be computed (piecewise geodesic with fewer breaks) than those in the previous paragraph, which uses much less information about the topology of the configuration space.

The authors have used these results to develop a complete program for motion planning for closed chains that works in polynomial time ${ }^{1}$ independent of whether the topology is well understood. The trade off is that these paths may be quite far from optimal.

Closed $k$-chains are a special family of linkages. Over the years, quite a number of papers have been written that deal with aspects of the problem of determining the configuration spaces and moduli spaces of linkages. It is known, as was shown by Thurston (unpublished, but see [KM3]), that the complexity of the full subject is that of real algebraic geometry, though, as the results above show, the situation becomes much more manageable when we restrict to special families. Recently the results of [KM1], [KM2], [KM3], provide a good review of previous work and give a number of interesting results on the structure of these configuration spaces, particularly the configuration spaces for closed chains in $\mathbb{R}^{2}$ and $\mathbb{R}^{3}$.

Worth special note is the work of J. C. Hausmann, also J. C. Housmann with A. Knutson [Ha], [HK] who determine the cohomology rings of a number of these spaces but not their detailed homotopy types. Also one should note the work of Y. Kamiyama and a number of collaborators [K1], [K2], [K3], [KTT], [KT] who study the case where all the edge lengths or all the edge lengths but one are the same by different methods.

In further work, the authors discuss extensions of the results above to configuration spaces for closed chains in the presence of obstacles and constraints. Also, we would like to thank Steven Kaufman for the help and encouragement he gave us throughout the development of these results.

\section{Background}

Kinematics is the study of the possible motions of systems of bodies coupled mechanically through contact constraints. These constraints can be permanent, as in the case of a hinge joint, or intermittent, as in the case of a ratchet mechanism. A common problem in mechanism design is to choose the number of links and their lengths, twists, and offsets, so as to allow a particular link to move (relative to a given base link) from one configuration to another, possibly following some specified rigid body motion. Currently, this design problem is solved taking little or no advantage of the structure of the space of configurations of the mechanisms under consideration. While some research results that leverage global structure of the configuration spaces have appeared in the literature, ${ }^{2}$ common design practices still tend to rely on iterative numerical procedures that use only local information.

${ }^{1}$ Here complete means that if it is possible to find a path from the initial configuration to the final configuration, the program will construct one, and if it is not possible, the program will report this as well.

${ }^{2}$ For example, Shukla and Mallik, [SM], developed a method to determine the existence of a crank (a link that can rotate $360^{\circ}$ relative to some other link in Watt and Stephenson chains (six-bar, planar mechanisms with two loops)). 
As a result, the design process for mechanisms with even small numbers of joints is tedious.

In the design of common one-degree-of-freedom mechanisms, such as the four-bar linkage and crank and slider mechanisms, $[\mathrm{H}]$, current design tools are reasonably powerful and efficient. However, the field of robotics has been placing increasingly difficult demands on mechanism designers. Most robotic applications require more degrees of freedom from mechanisms than current design tools can readily handle. One challenging class of robotics problems requires the motion planning and control of a closed-chain mechanism with many degrees of freedom. For example, a bombdisposal robot must be capable of moving to a door (behind which is a bomb), and opening it. While the robot is opening the door, a closed kinematic chain is formed that is composed of the robot arm and the door, connected to the ground at either end. To open the door, one must understand the constraints imposed on the system by the kinematic loop and be able to plan the motion of the system from an initial state (the door is closed) to a goal state (the door is fully open).

Despite the fact that bomb-disposal and many other robotic tasks require good designs and motion planning for closed kinematic chains, the state of the art is surprisingly crude. The most effective robot motion planners today are built upon randomized search techniques. [KLOS], [WXH]. However, individual randomized techniques have wildly varying performance and are not complete; they are not guaranteed to find a solution when one exists, nor can they determine that a solution does not exist when that is the case. The theoretical basis for a complete general motion planner was developed roughly 15 years ago, $[\mathrm{C}]$, but it has never been implemented due to the complexity of the specified algorithms.

The work presented here represents a first step in the development of maximally efficient, complete motion planners for robotic mechanisms. More importantly, the work expands the field of theoretical kinematics. Previously, the only mechanisms for which the global properties of configuration space were understood, were those of planar mechanisms with very small numbers of joints (e.g., the four-bar mechanism). Here we completely determine the global structure of configuration spaces of spatial $n$-bar mechanisms, where $n$ is arbitrary. The class of mechanisms considered are those forming a single closed loop. For planar mechanisms, all joints are of the type known as "revolute" (i.e., hinge joints); they constrain adjacent links in the loop allowing only relative rotation about the axis of the joint. For spatial mechanisms, all links are connected by "U"-joints (i.e., pairs of revolute joints with intersecting axes). In addition, one link is allowed to change its length (i.e., the mechanism may have one prismatic joint). While our analysis allows self-intersection of the links, once the associated configuration space is understood, there are standard methods in topology for dealing with restrictions on the embeddings so that, for example, there are no self-intersections or the mechanisms do not intersect given closed sets in $\mathbb{R}^{2}$ or $\mathbb{R}^{3}$. We will not discuss these techniques here, but will do so in subsequent work. 


\section{Planning Paths in the Configuration Space}

Assume that we are given two points, $A$ and $B$, in the configuration space of a closed chain in $\mathbb{R}^{n}$, with the last edge based at $\overrightarrow{0}$ and lying on the $x_{1}$-axis. Then the space of paths from $A$ to $B$ is homotopy equivalent to the loop-space $\Omega\left(\mathcal{B}\left(l_{1}, \ldots, l_{k}\right)\right)$ (if $A$ and $B$ lie in the same path-component) or it is empty. Consequently, for $k>4$ and $n=2, k>3$ and $n=3$, or $k>2$ for $n>3$, if the loop space is not empty, then there are many ways of moving from $A$ to $B$. Given the non-uniqueness of paths and the huge difficulty, in general, of determining geodesics between $A$ and $B$, one must identify the most important path attributes to guide their construction.

If any path between $A$ and $B$ will do, then one may proceed a step at a time. Using 1.6, we can check whether we are dealing with a path connected space or one that has two components. If there are two components, then they are distinguished by the relative positions of the three long links. For example, if the long links are $l_{2}, l_{3}$ and $l_{4}$ (as in Figure 5), then $P_{3}$ will be in one half-plane or the other relative to $l_{4}$.

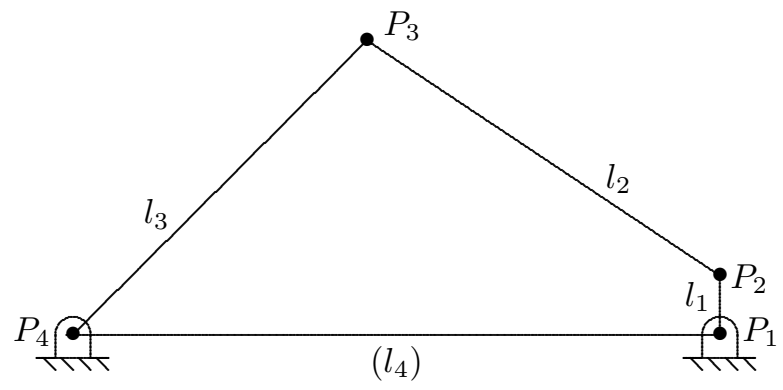

Figure 5: A four-bar mechanism with three long links.

In the two components case, (see 1.6), the configuration space is comprised of two tori and the short links are free to move without constraints. Hence the motion planning algorithm here is very simple: determine if $A$ and $B$ are in the same component and, if so, move the short links in a straight line on the universal cover of the torus from their configuration for $A$ to their configuration for $B$.

In the case of a single path connected component, one can simply move one link after another into the correct position, and then fix it. Having fixed a link, we can lump it with the old base link to form a new base link leaving a closed chain with one less link. The next move will be from the configuration just achieved, $A_{k}$, to the original goal configuration, $B$. Before beginning the next move, however, one checks the number of components in configuration space of the reduced chain. If there are two components and $A_{k}$ and $B$ are in the same component, proceed as in the previous paragraph. If they are not in the same component, we adjust the previous move to ensure that the long links move into the correct relative position before moving the next link into its correct final position.

Such algorithms take advantage only of our knowledge of the path components and our ability to detect which component contains a given configuration. But we also know much more about the geometry and topology of the configuration space 
than just the components. It turns out that the tori $\left(S^{n-1}\right)^{s} \times p t \subset\left(S^{n-1}\right)^{s} \times$ $I^{(n-1)(k-s-2)}$ in our $W$ 's are very close to geodesic, so when design constraints permit, it is quite efficient to locate one of these tori close to $A$, another close to $B$. This done, one can plan the path by constructing a path in the poset of the $\left(S^{n-1}\right)^{s}$ from the first torus to the other. Of course, this requires that one do a potentially very long analysis of a certain set of critical radii given explicitly in the statement of 1.12 and in more detail in 5.1. Algorithms for doing this can be extracted from the discussion that follows 7.8.

\section{Constructing Configuration Spaces of Closed Chains}

In this section we restrict ourselves to $\mathbb{R}^{2}$. It is direct to extend the discussion to $\mathbb{R}^{n}$ however, and we indicate the key changes as we proceed. Also, before we do the analysis of the closed situation, we consider open chains (where one end-point is allowed to vary but the other is fixed).

For open chains the structure of the configuration space is clear: a chain's configuration is determined by the successive angles between the edges, and between the base edge and some fixed ray emanating from the base-point. Consequently, the configuration space of an open chain with $k$ segments is just the $k$-torus $\left(S^{1}\right)^{k}$, (for $\mathbb{R}^{n}$, the product $\left.\left(S^{n-1}\right)^{k}\right)$.

We also need to consider the workspace of an open chain. This consists of all the points in the plane that occur as the image of the free end-point of the chain.

1. In the case of an open chain with a single edge of length $l$, the workspace is just the circle of radius $l$ centered at the base-point. (In $\mathbb{R}^{n}$ it is the sphere $S^{n-1}$ of radius $l$ centered at the base-point.)

2. In the case of an open chain with two unequal edges the workspace is always an annulus centered at the fixed end-point, with outer circle of radius $l_{1}+l_{2}$ and inner circle of radius $\left|l_{1}-l_{2}\right|$. (In $\mathbb{R}^{n}$ it is a product $S^{n-1} \times I$ having the same inner and outer radii as in $\mathbb{R}^{2}$.)

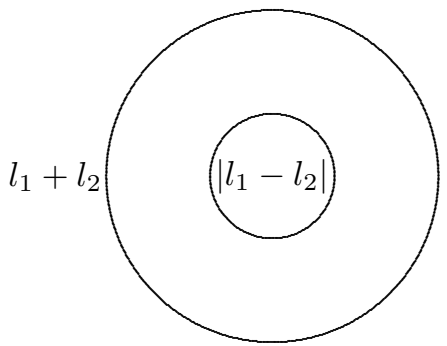

Figure 6: Annular Workspace for Two-Edge Linkage

Here, it is also worth noting that there are exactly two configurations with a given end-point as long as the end-point is in the interior of the annulus, while the configurations on the boundary circles occur only when the two edges lie on a single line through the base-point. In the case where the two edges have equal length the workspace is the entire disk of radius $2 l_{1}$, but the inverse image of the base-point in the configuration space consists of an entire circle. 
3. The workspace for a general open chain with at least three links is either a closed annulus or a closed disk centered at the origin. In both cases the outer radius will be $\sum_{i} l_{i}$.

Let us consider the configuration space of closed chains with three segments, i.e., planar 4-bar mechanisms. To do this we consider simultaneously an open chain with one edge of length $l_{3}$ based at $P_{4}$ and an open chain with two edges of lengths $l_{1}$ and $l_{2}$, based at $P_{1}$. Assume, for the moment that $P_{1}$ and $P_{4}$ are further apart than $l_{1}+l_{2}+l_{3}$, so there is no configuration of the closed chain that connects $P_{1}$ and $P_{4}$. Then start moving $P_{4}$ towards $P_{1}$ till the edges of the workspaces touch as shown in Figure 7:

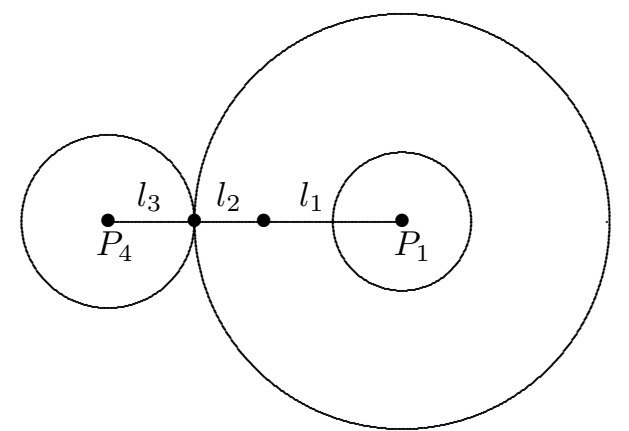

Figure 7: Workspaces Just Touching

Now there is a single solution - the three edges lie along the line containing $P_{1}$ and $P_{4}$. Continue to move $P_{4}$ towards $P_{1}$, so the intersection of the workspaces is an arc whose interior is completely contained in the interior of the annular workspace of the 2-chain. (In $\mathbb{R}^{n}, n \geqslant 3$, the intersection is a disc $D^{n-1} \subset S^{n-1}$.) At each interior point $\vec{v}$ of the arc there are exactly two configurations of the 2-chain at $P_{1}$ with $\vec{v}$ locating the free end-point. (In $\mathbb{R}^{n}, n \geqslant 3$ the configurations form an $S^{n-2}$ at each interior point of the $D^{n-1}$.) At each end-point of the arc there is only one configuration of the 2 -chain both in $\mathbb{R}^{2}$ and $\mathbb{R}^{n}$ with $n \geqslant 3$. Consequently, for the region defined by

$$
l_{1}+l_{2}>|| P_{1}-P_{4}||-l_{3}>\left|l_{1}-l_{2}\right|
$$

(provided that $\left\|P_{1}-P_{4}\right\|>l_{1}+l_{2}-l_{3}$ ) the configuration space is simply a circle for $n=2$, and $S^{n-1}$ more generally. (Four-bar mechanisms satisfying the condition that their configuration space is a single circle are referred to as non-Grashof in the engineering literature.)

The configuration space continues to be a circle, $\left(S^{n-1}\right)$, as $P_{4}$ moves towards $P_{1}$ until either the arc of intersection touches the interior circle of the annulus (or $P_{1}$ 
when the interior circle is degenerate, i.e., $\left.l_{2}=l_{1}\right)$, (which will occur if $2 l_{3}>\left|l_{1}-l_{2}\right|$ )

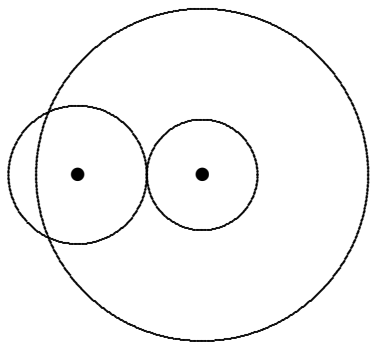

\section{Figure 8: Workspaces Just Touching at Inner Boundary}

or the intersection becomes the entire circle, with one point tangent to the outer circle of the annulus (which can only happen if $2 l_{3}<\left|l_{1}-l_{2}\right|$ ). Mechanisms with this type of configuration space are known in the engineering literature as uncertain since the inverse image of motion through the singular point given by three collinear links bifurcates.

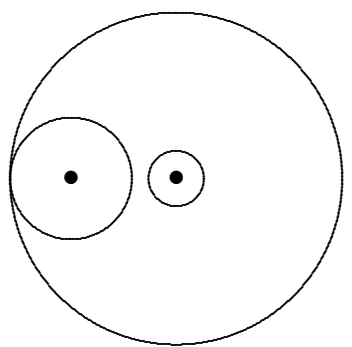

Figure 9: Workspace Contained and Touching Outer Boundary

In both these cases the configuration space becomes a figure 8 , while in the degenerate case (occurring when $2 l_{3}=\left|l_{1}-l_{2}\right|>0$ ), we find that the configuration space becomes the following graph:

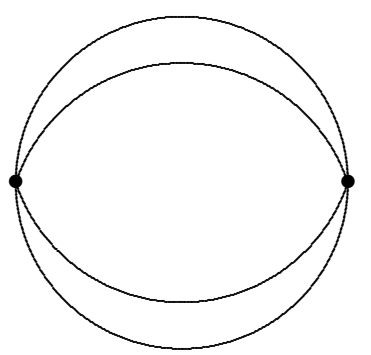

Figure 10: Three Loop Graph With Two Vertices

The remaining case occurs when the arc becomes tangent to the inner and outer circles of the annulus simultaneously and the inner circle degenerates to the point $P_{1}$, i.e., $l_{1}=l_{2}=l_{3}$. In this case the configuration space is a three vertex, six edge graph, with four edges incident on each vertex.

As $P_{4}$ continues to move towards $P_{1}$ various possibilities now occur. The two 
most important are represented in continuing the situations in both Figure 8 and Figure 9, where, in two different ways - as the circle crosses the inner radius in Figure 8 , or becomes entirely contained in the interior of the annulus for Figure 9 - the configuration space becomes two disjoint circles. In the engineering literature, this is referred to as Grashof, and represents the usual way in which four-bar mechanisms are applied. (Here is where the situation starts to significantly deviate between $\mathbb{R}^{2}$ and $\mathbb{R}^{n}, n \geqslant 3$. For example, the generic situation for $\mathbb{R}^{3}$ is the sequence $S^{3}, S^{1} \times$ $S^{2}, S^{3}, \mathbb{R P}^{3}$, while the corresponding sequence for $\mathbb{R}^{2}$ is $S^{1}, S^{1} \sqcup S^{1}, S^{1}, S^{1} \sqcup S^{1}$.)

The reader can easily list the remaining possibilities. A similar analysis can be done for five bar mechanisms in $\mathbb{R}^{2}$, and such an approach is discussed in [KM1]. There are exactly six non-singular closed surfaces that appear as configuration spaces for 5 -bar mechanisms in $\mathbb{R}^{2}$, the surfaces of genus $\leqslant 4$ and the disjoint union of two copies of the torus $S^{1} \times S^{1}$. However, as the number of bars increases or when $n \geqslant 3$, this approach becomes too complex, and we need more systematic and powerful methods. (This is already evident for the 4 -bar mechanisms in $\mathbb{R}^{n}$, $n \geqslant 3$ as the previous paragraph indicates.)

\section{Generic points for the map to the workspace}

The considerations above indicate that it should be possible to "bootstrap" from $k$-bars to $(k+1)$-bars, provided we understand the entire inverse map of the configuration space for the lengths $l_{1}, l_{2}, \ldots, l_{k-1}$ in the sense that we know the inverse images of all the points in the workspace. In doing the bootstrapping, we consider the intersection of the circle of radius $l_{k}$ and the workspace, assuming that the center of this circle is at distance $l_{k+1}$ from the origin. The configuration space of the $(k+1)$-bar is the inverse image of this intersection.

Additionally, the considerations above indicate that there are certain critical circles, (spheres $S^{n-1}$ for $\mathbb{R}^{n}$ ), in the workspace, those circles where the inverse image contains a configuration with all the edges collinear, and that the inverse image will be non-singular unless the circle of radius $l_{k}$ is tangent to one of these critical circles.

Both of these observations are true. In fact, even more is true. If we choose an initial point on the circle and take the (signed) distance on the component of the intersection of the circle of radius $l_{k}$ with the workspace that contains the initial point, as a function on the inverse image of the configuration space, then this function is locally Morse, with all its critical points contained in the inverse images of the intersection of this circle with the critical circles in the workspace.

Here are the basic results.

Theorem 5.1. Let $\vec{q}$ be any point in the workspace of the open chain with lengths

$$
\left\langle l_{1}, l_{2}, \ldots, l_{k}\right\rangle,
$$

then the inverse image (in the torus $\left.\left(S^{n-1}\right)^{k}\right)$, of a point $p$ in the workspace is an $(n-1)(k-1)-1$ dimensional manifold if and only if $p$ is not on one of the spheres centered at the center of the workspace having radius $R_{I}=\left|\sum l_{i}-2 \sum_{j \in I} l_{j}\right|$ where $I \subset\{1,2, \ldots, n\}$ is any subset. 
Theorem 5.2. Suppose that $\gamma$ is a rectifiable curve embedded in the workspace and $\overrightarrow{0} \notin \gamma$. Then the inverse image of $\gamma$ is non-singular manifold if and only if $\gamma$ is transverse to each of the critical spheres that it intersects.

(Here the critical circles and spheres are the spheres described in Theorem 5.1.)

Corollary 5.3. Let $R_{I}$ and $R_{J}$ be adjacent critical radii, and $W(I, J)$ the open annulus (in $\mathbb{R}^{2}$ ) or open spherical shell (in $\mathbb{R}^{n}$ ) between them, then the map of the inverse image of $W(I, J)$ onto $W(I, J)$ is a fibration.

Remark. The exact structure of this fibration will be clarified in 6.5. There is an action of $S O_{n}$ on $W(I, J)$ defined in 6.3 with the key property 6.4 that enables us to define these fibrations as bundles associated to the restricted action of $S O_{n-1}$ on the space $e^{-1}(w)$ for some $w \in W(I, J)$. Such bundles are generically non-trivial, but are trivial over any curve in $W(I, J)$ for $n \geqslant 2$.

As a consequence, if the curve $\gamma$ lies entirely in one of the $W(I, J)$, then the inverse image of $\gamma$ is also a product $I \times V$ where $V$ is the inverse image of an arbitrary point in $W(I, J)$, and it follows that any two embedded $\gamma$ which lie entirely in $W(I, J)$ have diffeomorphic inverse images.

Theorem 5.4. Suppose that the curve $\gamma$ in the workspace satisfies the properties above for non-singularity of the inverse image. Then arc length on $\gamma$ is locally a Morse function on the inverse image, $W$, of $\gamma$, with critical points exactly the points in $W$ intersected with the critical spheres where all the edges are collinear.

In the next two sections we give the proofs.

\section{The proofs of the general position theorems above}

Lemma 6.1. The map e which sends an open chain with lengths $l_{1}, \ldots, l_{k}$, based at 0 to its endpoint has the following properties:

(a) It is real algebraic.

(b) The singular points of e are precisely the $(n-1)$-spheres $\left(\vec{X}_{1}, \vec{X}_{2}, \ldots, \vec{X}_{k}\right)$ in the configuration space $\left(S^{n-1}\right)^{k}$ where all the $\vec{X}_{j}$ are parallel, (so $\vec{X}_{j}=$ $\left.(-1)^{\epsilon_{j}} \vec{X}_{1}, j=2, \ldots, k\right)$.

(c) e maps the singular set diffeomorphically onto a collection of spheres $S^{n-1}$ centered at the origin of radii $\left|l_{1}+\sum_{i=2}^{k}(-1)^{\epsilon_{i}} l_{i}\right|$ where the $(k-1)$-tuples $\left(\epsilon_{2}, \ldots, \epsilon_{k}\right)$ run over all the $2^{(k-1)}$ possibilities with each $\epsilon_{i} \in\{0,1\}$.

Example 6.2. If $\left(l_{1}, l_{2}, l_{3}, l_{4}\right)=(1,1.5,2,3)$ then there are exactly six critical radii, $.5,1.5,2.5,3.5,4.5$ and 7.5 with 1.5 and 2.5 occurring in four distinct ways, and each of the others in 2 distinct ways.

Proof. $S^{n-1}$ is real algebraic, so $\left(S^{n-1}\right)^{k}$ is as well. Moreover, the map $e$ is given explicitly as

$$
e\left(\vec{X}_{1}, \ldots, \vec{X}_{k}\right)=\sum_{1}^{k} l_{i} \vec{X}_{i}
$$


and is consequently real algebraic.

We now consider the tangent map $d(e)$. The tangent space to $\left(S^{n-1}\right)^{k}$ at $\left(\vec{X}_{1}, \ldots, \vec{X}_{k}\right)$ is the direct sum

$$
\tau_{\vec{X}_{1}}\left(S^{n-1}\right) \oplus \tau_{\vec{X}_{2}}\left(S^{n-1}\right) \oplus \cdots \oplus \tau_{\vec{X}_{k}}\left(S^{n-1}\right),
$$

and $d(e)$ is just $l_{i} \times I$ on $\tau_{\vec{X}_{i}}\left(S^{n-1}\right)$, where $I$ is the inclusion $\tau_{\vec{X}_{i}}\left(S^{n-1}\right) \subsetneq \tau_{\vec{X}_{i}}\left(\mathbb{R}^{n}\right)$ as the vector subspace $\vec{X}_{i}^{\perp}$. Clearly, since $\vec{X}_{i}^{\perp}$ has codimension 1 in $\mathbb{R}^{n}$, unless all these subspaces are the same, the subspace of

$$
\tau_{e\left(\vec{X}_{1}, \ldots, \vec{X}_{k}\right)}\left(\mathbb{R}^{n}\right)
$$

spanned by these subspaces will be $\tau_{e\left(\vec{X}_{1}, \ldots, \vec{X}_{k}\right)}\left(\mathbb{R}^{n}\right)$ itself, and the point will be nonsingular for $e$. But when all the images are the same, then $\vec{X}_{1}^{\perp}$ will be the image of $d(e)$ and the point will be singular.

Standard results for $C^{\infty}$ maps with compact inverse images of points show that $e$ is a fibration in any connected component of $e\left(\left(S^{n-1}\right)^{k}\right)-\operatorname{Im}(\operatorname{Sing}(e))$. Consequently this is the case for each annular region between two successive critical spheres. To understand these fibrations we need the action of $O_{n}$ on $\left(S^{n-1}\right)^{k}$ defined by

$$
g\left(\vec{X}_{1}, \ldots, \vec{X}_{k}\right)=\left(g\left(\vec{X}_{1}\right), \ldots, g\left(\vec{X}_{k}\right)\right),
$$

for $g \in O_{n}$. A key property of this action is that

$$
g\left(e\left(\vec{X}_{1}, \ldots, \vec{X}_{k}\right)\right)=e\left(g\left(\vec{X}_{1}\right), \ldots, g\left(\vec{X}_{k}\right)\right)
$$

so we have the commutative diagram

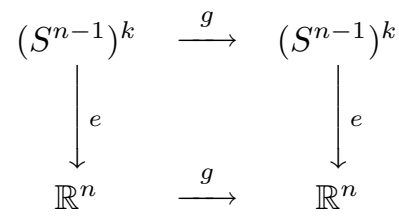

for each $g \in O_{n}$. As a consequence, if $e\left(\vec{X}_{1}, \ldots, \vec{X}_{k}\right)=\vec{Y}$ with $\vec{Y} \neq \overrightarrow{0}$ and $O_{n-1}(\vec{Y})$ is the subgroup of $O_{n}$ that fixes $\vec{Y}$. It follows that $O_{n-1}(\vec{Y})$ acts on $e^{-1}(\vec{Y})$ by restriction. Also, note that $O_{n-1}(\vec{Y})$ is conjugate to the usual $O_{n-1} \subsetneq O_{n}$.

As an example, when $k=2$ we know that for any point $\vec{Y}$ in the interior of the image of $e, e^{-1}(\vec{Y})=S^{n-2}$, and $O_{n-1}$ acts in the usual way on $S^{n-2} \subsetneq \mathbb{R}^{n-1}$. In this case, the fibration is given as the associated fiber bundle with total space

$$
S^{n-2} \times_{O_{n-1}} O_{n} \cong S^{n-2} \times_{S O_{n-1}} S O_{n}
$$

and projection

$$
\pi: S^{n-2} \times_{S O_{n-1}} S O_{n} \longrightarrow S O_{n-1} \backslash S O_{n}=S^{n-1}
$$

when restricted to any orbit sphere $S^{n-1}$ of $O_{n}$ in $V$. This is the the tangent bundle to $S^{n-1}$ and is non-trivial for $n \neq 2,4,8,[\mathrm{~A}]$. 
More generally, if $N=e^{-1}(\vec{Y})$ for $\vec{Y} \neq 0$ with $\vec{Y} \in \operatorname{Im}(e)$, and $k, n$ arbitrary, then we can describe $e^{-1}(V)$ as the product bundle

$$
I d \times \pi: I \times\left(N \times_{S O_{n-1}} S O_{n}\right) \longrightarrow I \times\left(S O_{n-1} \backslash S O_{n}\right)=I \times S^{n-1}
$$

associated to the $S O_{n-1}$ action on $N$.

Corollary 6.5. Let $V$ be the open annular region between two successive critical spheres in the situation above. Then the inverse image of $V$ is a fibration over $V$. The fibration is trivial, $V \times e^{-1}(V)$ if $n=2$, but is usually non-trivial for $n \geqslant 3$.

Let $\gamma$ be any rectifiable curve embedded in the workspace, and suppose that $W$ is the inverse image of $\gamma$ under $e$. Thus $W$ is generally a manifold of dimension $(n-1)(k-1)$ with boundary the disjoint union of the two manifolds $e^{-1}(\gamma(0))$ and $e^{-1}(\gamma(1))$. More precisely we have

Corollary 6.6. Suppose that $\gamma$ does not contain $\overrightarrow{0}$. Then $W$ is a differentiable manifold if and only if $\gamma$ intersects each critical sphere transversally. Moreover, if $\gamma$ satisfies these assumptions, then the set of critical points of the composition of the length function on $\gamma$ with $e$ on $W$ is exactly the set of $\left(\vec{X}_{1}, \ldots, \vec{X}_{n-1}\right) \in W$ where all the $\vec{X}_{i}$ are collinear.

Proof. Under these assumptions the map $e$ is transverse regular at every point of $\gamma$, and this implies that the inverse image of $\gamma, e^{-1}(\gamma)=W$, is a differentiable manifold.

It is clear that de restricted to $\tau_{v}(W)$ is onto $\tau_{e(v)}(\gamma)$ except possibly when $v$ is contained in the singular set of $e$. What needs to be checked is that de : $\tau_{v}(W) \rightarrow \tau_{e(v)}(\gamma)$ is the 0 -map whenever $v \in \operatorname{Sing}(e) \cap W$. But since $\gamma$ is transverse to $\operatorname{Im}(\operatorname{Sing}(e))$ at such a $v$, and the image of $d e\left(\tau_{v}\left(\left(S^{n-1}\right)^{i}\right) \subset \tau_{e(v)}(\operatorname{Im}(\operatorname{Sing}(e)))\right.$, it follows that the image of $d e \mid \tau_{v}(W)$ must be $\overrightarrow{0}$ in $\tau_{e(v)}(\gamma)$.

Thus we have shown that under the assumptions above, the length function $s_{\gamma} f$ restricted to $W$ has isolated critical points. It remains to analyze the Hessian at these critical points to prove that this function is Morse.

A key example: The curve we will study now is $\gamma=\left(\begin{array}{c}t \\ 0 \\ \vdots \\ 0\end{array}\right)$ in a neighborhood of the singular image $\left(\begin{array}{c}\left|\sum_{1}^{k}(-1)^{\epsilon_{i}} l_{i}\right| \\ 0 \\ \vdots \\ 0\end{array}\right)$. Let $W$ be the inverse image of $\gamma$ for $t$ in the interval

$$
\left(\left|\sum_{1}^{n}(-1)^{\epsilon_{i}} l_{i}\right|-\delta,\left|\sum_{1}^{n}(-1)^{\epsilon_{i}} l_{i}\right|+\delta\right)
$$

with $\delta>0$ appropriately small. On this $W$ we have that the arc-length function is simply the first coordinate of $\gamma, t$ up to adding an appropriate constant. 
In a neighborhood of the critical point in $W$ we have the following points in the configuration space

$$
\left.(-1)^{\epsilon_{1}}\left(\begin{array}{c}
\sqrt{1-m_{12}^{2}-\cdots-m_{1 n}^{2}} \\
m_{12} \\
\vdots \\
m_{1 n}
\end{array}\right), \cdots,(-1)^{\epsilon_{k}}\left(\begin{array}{c}
\sqrt{1-m_{k 2}^{2}-\cdots-m_{k n}^{2}} \\
m_{k 2} \\
\vdots \\
m_{k n}
\end{array}\right)\right)
$$

and the constraints that they lie in $W$ are $\sum_{1}^{k} l_{j} m_{j s}=0$ for $2 \leqslant s \leqslant n$. It follows that we can take the $m_{j s}, 2 \leqslant s \leqslant n, 2 \leqslant j \leqslant k$ as local coordinates in $W$ near the critical point. In terms of these coordinates, the terms $m_{12}, \ldots, m_{1 n}$ are given by

$$
m_{1 i}=(-1)^{\epsilon_{1}+1} \sum_{2}^{k}(-1)^{\epsilon_{j}} \frac{l_{j}}{l_{1}} m_{j i}, \text { for } 2 \leqslant i \leqslant n .
$$

Also, the arc-length function is given in the form

$$
f\left(m_{22}, \ldots, m_{k n}\right)=\sum_{i=1}^{k}(-1)^{\epsilon_{i}} l_{i} \sqrt{1-m_{i 2}^{2}-\cdots-m_{i n}^{2}},
$$

up to adding an appropriate constant.

The partial derivatives are

$$
\begin{aligned}
\frac{\partial f}{\partial m_{i j}} & =(-1)^{\epsilon_{1}+1} l_{1} \frac{m_{1 j}}{\sqrt{1-m_{12}^{2}-\cdots-m_{1 n}^{2}}} \frac{\partial m_{1 j}}{\partial m_{i j}}+(-1)^{\epsilon_{i}+1} l_{i} \frac{m_{i j}}{\sqrt{1-m_{i 2}^{2}-\cdots-m_{i n}^{2}}} \\
& =(-1)^{\epsilon_{i}} \frac{m_{1 j}}{\sqrt{1-m_{12}^{2}-\cdots-m_{1 n}^{2}}} l_{i}+(-1)^{\epsilon_{i}+1} l_{i} \frac{m_{i j}}{\sqrt{1-m_{i 2}^{2}-\cdots-m_{i n}^{2}}}
\end{aligned}
$$

and they are, as expected, all 0 at the point $m_{i j}=0,2 \leqslant i \leqslant k, 2 \leqslant j \leqslant n$. Similarly, the second derivatives $\frac{\partial^{2} f}{\partial m_{i j} \partial m_{v w}}$ are 0 unless $w=j$, and

$$
\frac{\partial^{2} f}{\partial m_{i j} \partial m_{v j}}=(-1)^{\epsilon_{1}+\epsilon_{i}+\epsilon_{v}+1} \frac{l_{i} l_{v}}{l_{1}}+\delta_{v, i}(-1)^{\epsilon_{i}+1} l_{i}
$$

in the case where $w=j$. Here $\delta_{v, i}=\left\{\begin{array}{ll}0 & \text { if } v \neq i \\ 1 & \text { if } v=i .\end{array}\right.$. Thus the Hessian matrix, $H$, of second partial derivatives is a block diagonal matrix of the form

$$
H=\left(\begin{array}{ccccc}
D & 0 & 0 & \ldots & 0 \\
0 & D & 0 & \ldots & 0 \\
0 & 0 & D & \ldots & 0 \\
\vdots & \vdots & \vdots & \ddots & \vdots \\
0 & 0 & 0 & \ldots & D
\end{array}\right)
$$

with $D$ a $(k-1) \times(k-1)$ matrix and $(-1)^{\epsilon_{1}+1} l_{1}$ multiplied by the $i-1, v-1$ term of $D$ equal to

$$
(-1)^{\epsilon_{i}+\epsilon_{v}} l_{i} l_{v}+\delta_{i, v}(-1)^{\epsilon_{1}+\epsilon_{i}} l_{i} l_{1}
$$


Consequently, $D$ is the sum of the diagonal matrix

$$
C=(-1)^{\epsilon_{1}+1} \frac{1}{l_{1}}\left(\begin{array}{ccccc}
(-1)^{\epsilon_{2}+\epsilon_{1}} l_{1} l_{2} & 0 & 0 & \ldots & 0 \\
0 & (-1)^{j(3)} l_{1} l_{3} & 0 & \ldots & 0 \\
0 & 0 & (-1)^{j(4)} l_{1} l_{4} & \ldots & 0 \\
\vdots & \vdots & \vdots & \ddots & \vdots \\
0 & 0 & 0 & \ldots & (-1)^{\epsilon_{k}+\epsilon_{1}} l_{1} l_{k}
\end{array}\right)
$$

and the rank 1 matrix

$$
R R^{t}=(-1)^{\epsilon_{1}+1} \frac{1}{l_{1}}\left(\begin{array}{cccc}
l_{2}^{2} & (-1)^{\epsilon_{2}+\epsilon_{3}} l_{2} l_{3} & \ldots & (-1)^{\epsilon_{2}+\epsilon_{k}} l_{2} l_{k} \\
(-1)^{\epsilon_{2}+\epsilon_{3}} l_{2} l_{3} & l_{3}^{2} & \ldots & (-1)^{\epsilon_{3}+\epsilon_{k}} l_{3} l_{k} \\
\vdots & \vdots & \ddots & \vdots \\
(-1)^{\epsilon_{2}+\epsilon_{k}} l_{2} l_{k} & (-1)^{\epsilon_{3}+\epsilon_{k}} l_{3} l_{k} & \ldots & l_{k}^{2}
\end{array}\right)
$$

where $R^{t}=\left((-1)^{\epsilon_{2}} l_{2}, \ldots,(-1)^{\epsilon_{k}} l_{k}\right)$. Since $R R^{t}$ is rank 1 , it follows that all the $s \times s$ minors of $R R^{t}$ are 0 for $k-1 \geqslant s \geqslant 2$. From this it is direct that

$$
\operatorname{Det}(D)= \pm \frac{1}{l_{1}} \prod_{2}^{k} l_{j} \times\left(\sum_{1}^{k}(-1)^{\epsilon_{j}} l_{j}\right)
$$

is non-zero. We have verified that $f$ is Morse on this special $\gamma$.

We now complete the proofs of our main theorems by showing that this special case implies the general case.

Let $v \in W \cap \operatorname{Sing}(e)$ have image $\mu$ contained in the sphere of radius $\sum_{1}^{k}(-1)^{\epsilon_{i}} l_{i}$ about $\overrightarrow{0}$. Let $\mu \in \gamma=e(v)$. Then there is an open neighborhood of $e(v)$ in $\gamma, N$, so that

(1) at most one point in $N$ has distance $t$ from the origin (from the fact that $N$ is transverse to the sphere of radius $\left.\sum_{1}^{k}(-1)^{\epsilon_{i}} l_{i}\right)$,

(2) there is a $C^{\infty}$ map $\lambda: N \rightarrow S O_{n}$ so that for each $v \in N, \lambda(v)(v)$ is contained in the curve $\left(\begin{array}{c}t \\ 0 \\ \vdots \\ 0\end{array}\right)$ above.

The corresponding action of $\lambda(N)$ on $e^{-1}(N)=W_{1}$, (defined in 6.3), gives a diffeomorphism from $e^{-1}(N)$ to $e^{-1}(\lambda(N))$, (because of 6.4), and the original arc-length function on $N$ is a composition $h \circ f \circ \lambda$ with $\frac{d h}{d t} \geqslant 1$ at the image of $v$. But from this, the Hessian with respect to arc-length on $\gamma$ is the same as the Hessian on the sample curve above multiplied by $\frac{d h}{d t}$ evaluated at $\mu(e(v))$. This completes the proof of our main technical assertion, Theorem 5.4. 


\section{The structure of inverse images of curves}

It turns out that we do not need the exact index of these critical points. What matters is how many of them there are in the inverse image of a given path in the workspace. But before we get into these details we need to make a few remarks about the dependence of these inverse images on the particular path, $\gamma$.

Throughout this section we assume that the inequalities among the $l_{1}, \ldots, l_{n}$ required for the non-singularity of all the critical points are satisfied.

By assumption $\gamma$ is differentiable and has transverse intersections with the critical point spheres of the map from the free configuration space to the workspace. Assume that $\gamma$ has been parameterized by (scaled) arc-length and is thus given by a unique differentiable map $I \longrightarrow e\left(W\left(l_{1}, \ldots, l_{n}\right)\right)$. It follows that the union of these intersection points forms a discrete labeled configuration of points in $I$, where the labeling is by the radius of the particular critical sphere containing the image. Clearly, there is a single constraint on this set - adjacent labeled points must either be labeled by the same radius or by the next larger or smaller radius. But aside from this constraint any finite, discrete configuration of labeled points can arise. We call the resulting labeled configurations that actually arise admissible configurations. All the admissible configurations are naturally ordered via the natural ordering of the inverse images of the critical spheres in $I$, and thus, associated to each admissible configuration there is a unique ordered sequence of radii of critical spheres.

Definition 7.1. Two admissible configurations are equivalent if and only if the associated ordered sequences of radii of critical spheres are equal.

The following result is now direct from 5.3.

Theorem 7.2. Let $\gamma_{1}$ and $\gamma_{2}$ be two admissible curves in the workspace $W\left(l_{1}, \ldots, l_{n}\right)$ with the same end-points which are not on the critical spheres. Then the inverse images of the two curves are diffeomorphic if their associated configurations of labeled critical points are equivalent.

Remark 7.3. It is clear that one does not actually need the endpoints of the two curves to be equal, merely that they lie in the interiors of the same annular regions between adjacent critical radii.

Definition 7.4. A curve $\gamma$ is monotone if the associated ordered sequence of critical radii is monotone.

In the case of monotone curves, in order to understand the diffeomorphism type of the associated inverse image, it is sufficient to assume that the curve is a segment of a ray from the origin, and we will concentrate on monotone curves - and consequently, segments on rays from the origin - in what follows.

Example 7.5. Suppose that the base is a prismatic joint, which, for simplicity, we will assume simply means that the length of the base varies in the closed interval $\left[l_{n}(0), l_{k}(1)\right]$. Then the resulting configuration space will be the inverse image of the line segment

$$
\left[l_{k}(0), l_{k}(1)\right]
$$


along the positive $x$-axis.

Example 7.6. Suppose that we are interested in the inverse image of $x_{k}$ with $0<x_{k}<\sum_{1}^{k-1} l_{i}$ along the $x$-axis, and suppose that $x_{k}$ is not a critical radius. Then the configuration space $\mathcal{C}\left(l_{1}, \ldots, l_{k-1}, x_{k}\right)$ is non-singular and is the boundary of the inverse image of the segment $\left[x_{k}, l_{1}+l_{2}+\cdots l_{k-1}\right]$.

Likewise, the union of the configuration spaces $\mathcal{C}\left(l_{1}, \ldots, l_{k}(0)\right)$ and $\mathcal{C}\left(l_{1}, \ldots, l_{k}(1)\right)$ is the boundary of the configuration space described in 7.5.

Example 7.7. When we bootstrap in the plane, and construct the configuration space

$$
\mathcal{C}\left(l_{1}, \ldots, l_{k}\right)
$$

by taking the inverse image of the intersection of the circle centered at $l_{k}$ of radius $l_{1}$ with the free workspace for $\left(l_{2}, \ldots, l_{k-1}\right)$ we break the intersection up into two pieces, the inverse image of the part of the circle above the $x$-axis and the inverse image of the part of the circle below it. Both of these are monotone and consequently diffeomorphic. We have just proved the following theorem:

Theorem 7.8. The configuration space of a closed chain in $\mathbb{R}^{2}$ is the double over the boundary of the inverse image of a monotone path and hence a segment along the $x$-axis.

Remark. Bootstrapping in $\mathbb{R}^{n}$ involves the inverse image of the intersection of an $(n-1)$-sphere, $S^{n-1}$, with the workspace. This inverse image also breaks up into a union over the common boundary of two diffeomorphic pieces, one corresponding to the upper hemisphere and one to the lower. But our current techniques only enable us to identify the inverse images of curves $\gamma$ and so the exact structure of these inverse images for $n>2$ are not really available in this way. However, by using curves in the image of the configuration space for all $k$ links we are able to determine these spaces explicitly, though this description does not give them as explicit doubles.

\section{The homotopy types of the inverse images of curves}

In $\S 7$ we reduced the analysis of the inverse image of any $C^{\infty}$ curve in the workspace to the study of the inverse images of straight line segments. In this section we consider the inverse image of the line segment $S$ along the $x_{1}$-axis from some $l>0$ to $\sum l_{i}$, the radius of the workspace, where $l$ is not a critical value. For convenience let $E_{x}$ be the unit vector pointing in the positive $x_{1}$-direction in $S$.

$$
E_{x}=\left(\begin{array}{c}
1 \\
0 \\
\vdots \\
0
\end{array}\right) .
$$


Suppose that $r_{I} \in S$, is a critical radius. Note that if $I^{\prime}$ is the complement of $I$ - the set of $j, 1 \leqslant j \leqslant k$ with $j \notin I$ - then $r_{I^{\prime}}$ is equal to $r_{I}$, so we generally have two different critical points for the same critical radius. To choose between them we note that

$$
\sum_{i \in I}-l_{i}+\sum_{j \notin I} l_{j}=-\left(\sum_{s \in I^{\prime}}-l_{s}+\sum_{w \notin I^{\prime}} l_{w}\right) \neq 0
$$

so only one choice of signs gives a positive signed sum. In the rest of this section, we assume that the signed sum for $I$ is the positive one. Consequently, for each $I$ with critical radius $r_{I}$ contained in $S$ we have.

$$
\sum_{i \notin I} l_{i}-\sum_{j \in I} l_{j}=r_{I}>0
$$

For each $j \in I$, the $j$-interval is parallel to the $x_{1}$-axis and points inward.

For each such $I$ we denote by $T^{I}$ the subspace $\left(S^{n-1}\right)^{|I|}$ contained in the configuration space defined by setting the unit vectors which are not in $I$ to be $E_{x}$, while freely varying the links with $i \in I$. The image of the endpoint map $e$ on $T^{I}$ is an annulus or an $n$-ball centered about the point $\sum_{i \notin I} l_{i} E_{x}$ having outer radius $\sum_{i \in I} l_{i}$.

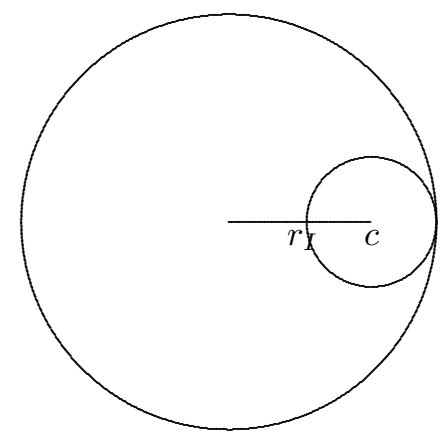

Figure 11: $c=\sum_{i \notin I} l_{i}$

Thus, to each critical value $r_{I}$ in $S$ there is associated a unique sub-coordinate product $\left(S^{n-1}\right)^{|I|}$ in the total configuration space $\left(S^{n-1}\right)^{k}$, and these $T^{I}$ fit together in the sense that if $J \subset I$, then $r_{J} \in S$ and $T^{J} \subset T^{I}$. Conversely, for each coordinate sub-product $\left(S^{n-1}\right)^{m}$ contained in $T^{I}$, there is a unique $J$ so this sub-product is $T^{J}$.

Definition 8.2. The union of these $T^{I}, r_{I} \in S$, is written $X_{S}$.

As constructed, $X_{S}$ does not lie in $e^{-1}(S)$. In fact, all that we can say about $X_{S}$ at this point is that

(8.3) $e\left(X_{S}\right)$ is entirely contained in the positive half-ball of the workspace, that is to say, the region consisting of all points with positive first coordinate, $x_{1}>0$.

(8.4) The integral homology of $X_{S}$ is torsion free, has one generator for each $r_{I} \in S$ 
(in dimension $|I|(n-1)$ ) and injects into the homology of the entire configuration space.

(For 8.4 note that $X_{S}$ is a sub-complex of the $C W$-complex $\left(S^{n-1}\right)^{k}$ where $S^{n-1}$ is given as a cell complex having a single 0-cell, $e_{0}=E_{x}$, and a single $(n-1)$-cell, while $\left(S^{n-1}\right)^{k}$ is realized as the product $C W$-complex. We now indicate how to construct a deformation of $X_{S}$ to a sub-complex of $e^{-1}(S)$.)

Recall the definition of the action $S O_{n} \times\left(S^{n-1}\right)^{k} \rightarrow\left(S^{n-1}\right)^{k}$ defined in 6.3.

Lemma 8.5. There is a continuous map $h: I \times X_{S} \rightarrow S O_{n}$ satisfying $h \mid 0 \times X_{S}$ is the identity in $S O_{n}$, so that the one parameter family of embeddings

$$
P_{t}: X_{S} \longrightarrow\left(S^{n-1}\right)^{k}
$$

defined by $P_{t}(v)=h(t, v)(v)$, satisfies $P_{1}(v) \in e^{-1}(S)$ for all $v \in X_{S}$.

Proof. The fibration,

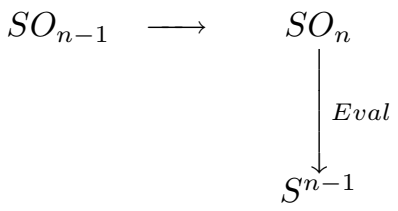

where $\operatorname{Eval}(g)=g\left(E_{x}\right)$ for $g \in S O_{n}$, is trivial over $S^{n-1}-\left(-E_{x}\right)$. Consequently, there is a $C^{\infty}$ lifting $L: S^{n-1}-\left(-E_{x}\right) \rightarrow S O_{n}$ so that

(1) $\mathrm{Eval} \cdot L=I d$,

(2) $L\left(E_{x}\right)=I d \in S O_{n}$.

For an explicit lifting see, e.g., [ST].

For any point $v \in X_{S}, e(v)$ is in the positive half-ball, and, in particular $e(v) \neq 0$. The unit vector from the origin pointing in the direction of $e(v)$ consequently lies in $S^{n-1}-\left(-E_{x_{1}}\right)$. Moreover, there is a unique geodesic in $S^{n-1}-\left(-E_{x_{1}}\right)$ between this unit vector and $E_{x_{1}}$. Using these geodesics we obtain, in the obvious way, a map $\lambda: I \times X_{S} \rightarrow S^{n-1}-\left(-E_{x_{1}}\right)$, so that $\lambda(1, v)$ is the unit vector in the direction of $e(v)$ and $\lambda(0, v)=E_{x_{1}}$.

The map $h$ in 8.5 is now defined as

$$
h(t, v)=(L \circ \lambda(t, v))^{-1} .
$$

Corollary 8.7. $e^{-1}(S)$ is homotopy equivalent to $X_{S}$ and is tautly embedded in $\left(S^{n-1}\right)^{k}$ in the sense that the integral homology of $e^{-1}(S)$ embeds into the integral homology of $\left(S^{n-1}\right)^{k}$ as a direct summand.

Proof. We have constructed an embedding of $X_{S}$ in $e^{-1}(S)$, and a homotopy of this embedding to a $C W$-embedding of $X_{S}$ as a sub-complex of the usual cellular decomposition of $\left(S^{n-1}\right)^{k}$. Moreover, the integral homology of $e^{-1}(S)$ generated by these cells forms a direct summand of $H_{*}\left(e^{-1}(S), \mathbb{Z}\right)$. Consequently, we have shown that every cell of $X_{S} \subsetneq e^{-1}(S)$ is essential. On the other hand, there are exactly as 
many cells in $X_{S}$ as there are critical points in $e^{-1}(S)$ for the Morse function discussed in 6.6. Hence, we have accounted for all the cells needed to construct $e^{-1}(S)$, and 8.7 follows. For $n>2$ it follows that there are no (1)-cells in the decomposition, and the fundamental group $\pi_{1}\left(e^{-1}(S)\right)=\{1\}$. Consequently, the homology isomorphism gives a homotopy equivalence using the Whitehead theorems. When $n=2$ we have to be a little more careful. Note that we can extend the embeddings constructed in this section to embeddings of the normal bundles to the products of spheres, $\left(S^{n-1}\right)^{l}$, as open neighborhoods in $e^{-1}(S)$. (In the next section we will show that these normal bundles are actually trivial but we don't need this here.) Since each of these products of spheres contains exactly as many critical points as the sum of the dimensions of the homology groups of $\left(S^{n-1}\right)^{l}$, the same is true for the embedded normal bundle provided we make it sufficiently small.

The Morse flow associated to the Morse function can be assumed to stay within this neighborhood in a sufficiently small neighborhood of the key critical point. Then, by induction, one can argue that the attaching map of this top cell is the usual one, attaching it as the top cell of the product of $S^{n-1}$-spheres. This completes the proof.

Remark 8.8. It follows from 8.7 that the cohomology map

$$
H^{*}\left(\left(S^{n-1}\right)^{k}, \mathbb{Z}\right) \rightarrow H^{*}\left(e^{-1}(S), \mathbb{Z}\right)
$$

is surjective (and explicit). Consequently, the cup-products in $H^{*}\left(e^{-1}(S), \mathbb{Z}\right)$ are entirely determined.

\section{The normal neighborhoods of the $T^{I} \subset e^{-1}(S)$.}

As we have noted in 8.3, the unit vector from the origin in the direction of any point in $e\left(X_{S}\right)$ always lies in the open right hemisphere. Hence, since $X_{S}$ is compact, there is an $\epsilon>0$ so that this statement remains true if, instead of taking

$$
\left(\sum_{j \notin I} l_{j}\right) E_{x}+\sum_{i \in I} l_{i} X_{i}
$$

to define our interior $n$-ball or annulus, we take the sum

$$
\left(\left(\sum_{j \notin I} l_{j}\right)-t\right) E_{x}+\sum_{i \in I} l_{i} X_{i}
$$

for $0 \leqslant t \leqslant \epsilon$ to define a new interior $n$-ball or annulus.

Now we restrict attention to just the links in the set $J=\{i \mid i \notin I\}$, and consider the end-point map on their configuration space $\left(S^{n-1}\right)^{|J|}$. Let $R=\sum_{j \in J} l_{j}$ be the radius of the image of this end-point map. Let $\gamma_{J} \subsetneq$ the $n$-ball of radius $R$ be the 
path

$$
\left(\begin{array}{c}
R-t \\
0 \\
0 \\
\vdots \\
0
\end{array}\right)
$$

for $0 \leqslant t<\epsilon$ with $\epsilon$ satisfying the condition above and also the condition that $\gamma$ only intersects the single singular image sphere of radius $R$. Since $e^{-1}\left(\gamma_{J}\right)$ contains only a single critical point, a maximum, it is a ball of dimension $(n-1)(|J|-1)$, $D^{(n-1)(|J|-1)}$. Consequently we have an explicit embedding

$$
h_{I}: M_{I}\left(T^{I}\right) \cong D^{(n-1)|J|} \times T^{I} \subset\left(S^{n-1}\right)^{k} .
$$

This embedded set has codimension $(n-1)$, so it is not open. But this is exactly the codimension of $e^{-1}(S)$ in $\left(S^{n-1}\right)^{k}$, so if it were to be contained in $e^{-1}(S)$ it would be open there.

Let $P_{I}$ the the composition $e \circ h_{I}$.

$$
P_{I}: D^{(n-1)(|J|-1)} \times S^{(n-1)|I|} \longrightarrow \mathbb{R}^{n} .
$$

It's image is contained entirely in the subspace of image $e$ with $x_{1} \geqslant \tau$ for a $\tau>0$. Consequently, the map $\pi_{I}$ from $\operatorname{Im}\left(P_{I}\right)$ to $S^{n-1}$ obtained by taking the unit vector in the direction of the line from the origin to $P_{I}(w)$ for each $w \in M_{I}\left(T^{I}\right)$, is contained in $S^{n-1}-\left(-E_{x}\right)$, and we have a well defined map

$$
\lambda: M_{I}\left(T^{I}\right) \longrightarrow S O_{n}
$$

defined as the composition $L \circ \pi_{I}$. As was the case with 8.5 we have

Lemma 9.3. The map

$$
\Lambda: M_{I}\left(T^{I}\right) \longrightarrow\left(S^{n-1}\right)^{k}
$$

defined by $\Lambda(w)=\lambda(w)^{-1} P_{I}(w)$ is a differentiable embedding $M_{I}\left(T_{I}\right) \subset e^{-1}(S)$.

This establishes the fact that the normal bundle to $T^{I}$ in $e^{-1}(S)$ is trivial. The next thing to check is how two different $T^{I}$ and $T^{I^{\prime}}$ intersect the normal bundle to $T^{I \cap I^{\prime}}$.

Lemma 9.4. Let $I^{\prime \prime}=I \cap I^{\prime}$, so we have

$$
T^{I} \cap T^{I^{\prime}}=T^{I^{\prime \prime}} .
$$

The following statements hold.

$9.5 I \cup I^{\prime} \neq\{1,2,3, \ldots, k\}$.

9.6 Let

- $K_{1}=I-\left\{I \cap I^{\prime}\right\}$,

- $K_{2}=I^{\prime}-\left\{I \cap I^{\prime}\right\}$,

- $K_{3}$ be the compliment of $I \cap I^{\prime}$ in $\{1,2, \ldots, k\}$. 
Then we can choose coordinates for the disk $e^{-1}\left(\gamma_{I \cap I^{\prime}}\right)$ in a sufficiently small neighborhood of the point where all the vectors are equal so that the coordinates corresponding to $K_{1} \cup K_{2}$ are part of the coordinate set.

For 9.5 note that $\sum_{1}^{k} l_{j}-2 \sum_{i \in I} l_{i}>0$ and $\sum_{1}^{k} l_{j}-2 \sum_{m \in I^{\prime}} l_{m}>0$. If $I \cup$ $I^{\prime}=\{1, \ldots, k\}$, then both the above equations cannot hold simultaneously. Thus $I \cup I^{\prime} \neq\{1, \ldots, k\}$.

For 9.6 note that 9.5 implies that $K_{1} \cap K_{2}=\emptyset$ and $K_{1} \cup K_{2} \subsetneq K_{3}$. Let $v \in$ $\{1, \ldots, k\}$ be contained in $K_{3}-\left(K_{1} \cup K_{2}\right)$. Let $\gamma$ be the curve 9.2 for $K_{3}$. Then $\gamma$ has codimension $(n-1)$ in the workspace so it is determined in a perhaps smaller neighborhood of the extremal point $\sum_{j \in K_{3}} l_{j} E_{x_{1}}$ by making an arbitrary single coordinate a function of the remaining coordinates. In particular, we can choose this coordinate to be the one corresponding to $v$. So 9.6 follows.

Let $K_{4}=\{1, \ldots, k\}-I$ and let $K_{5}=\{1, \ldots, k\}-I^{\prime}$. Both $T^{I}$ and $T^{I^{\prime}}$ contain $T^{I \cap I^{\prime}}$, but it is not true that $M_{I}\left(T^{I}\right)$ and $M_{I^{\prime}}\left(T^{I^{\prime}}\right)$ are contained in $M_{I \cap I^{\prime}}\left(T^{I \cap I^{\prime}}\right)$. We now want to discuss the way that the normal bundles to the two products $T^{I}$ and $T^{I^{\prime}}$ fit together in a neighborhood of $T^{I \cap I^{\prime}}$.

Theorem 9.7. Let $M_{I \cap I^{\prime}}\left(T^{I \cap I^{\prime}}\right)=T^{I \cap I^{\prime}} \times \mathbb{R}^{K_{1}} \times \mathbb{R}^{K_{2}} \times \mathbb{R}^{K_{4} \cap K_{5}}$ where the factors $\mathbb{R}^{K_{1}}$ and $\mathbb{R}^{K_{2}}$ are the coordinate planes of 9.6. Then the normal bundles $N_{T^{I}}\left(T^{I \times I^{\prime}}\right)$ and $N_{T^{I^{\prime}}}\left(T^{I \times I^{\prime}}\right)$ span $\mathbb{R}^{K_{3}} \times \mathbb{R}^{K_{4}}$ at every point of $T^{I \cap I^{\prime}}$.

Proof. Let $V$ be a small neighborhood of $T^{I \cap I^{\prime}}$ in $T^{I}$. Then the composition

$$
(\Lambda)^{-1} \circ P_{1}: V \longrightarrow M_{I \cap I^{\prime}}\left(T^{I \cap I^{\prime}}\right)
$$

defined using $\Lambda$ from 9.3 and $P_{1}$ from 8.5 is what one expects on $T^{I \cap I^{\prime}}$ but is not quite expected on $V-T^{I \cap I^{\prime}}$. Thus, on coordinates associated to $K_{1}$ the $X_{j}$ are close to $E_{x_{1}}$, but on $K_{4}$ coordinates, all the $X_{v}$ are equal, but not necessarily equal to $E_{x_{1}}$, since we must have

$$
\sum_{j \in K_{1}} l_{j} X_{j} \oplus\left(\sum_{v \in K_{4}} l_{v}\right) X_{v_{0}}=t E_{x_{1}},
$$

for some positive value of $t$. Thus we can give coordinates for this region as the $X_{j}$, $j \in K_{1}$, with the elements in $K_{2}$ and $K_{4}-K_{2}$ determined via the equations

$$
\sum_{i \in K_{1}} l_{i} X_{i}+\left(\sum_{j \in K_{4}} l_{j}\right) X_{v_{0}}=t E_{x_{1}} .
$$

Consequently, the $\tau$ coordinate in $X_{v_{0}}$ is given as

$$
\frac{-1}{\sum_{j \in K_{4}} l_{j}} \sum_{1 \in K_{1}} l_{i} X_{i, \tau}, 2 \leqslant \tau \leqslant n,
$$

and we have

$$
\frac{\partial X_{v_{0}, \tau}}{\partial X_{i, \tau}}=\frac{-l_{i}}{\sum_{j \in K_{4}} l_{j}} .
$$


Let $A_{1}=\sum_{i \in K_{1}} l_{i}, A_{2}=\sum_{j \in K_{2}} l_{j}$ and $A_{3}=\sum_{w \in K_{3}} l_{w}$. Then the Jacobian for the embeddings of the two normal bundles to $T^{I \cap I^{\prime}}$ in $T^{I}$ and $T^{I^{\prime}}$ has the form

$$
\left(\begin{array}{cc}
I & N \\
N^{\prime} & I
\end{array}\right)
$$

where the general form of $N$ is $(n-1)$ equal diagonal blocks, (one for each $\tau$ ). Each diagonal block has the form

$$
N=\frac{-1}{A_{2}+A_{3}}\left(\begin{array}{cccc}
l_{i_{1}} & l_{i_{1}} & \ldots & l_{i_{1}} \\
\vdots & \vdots & \ddots & \vdots \\
l_{i_{r}} & l_{i_{r}} & \ldots & l_{i_{r}}
\end{array}\right)
$$

where $i_{1}, \ldots i_{r}$ are the coordinates in $K_{1}$. There is a similar description for $N^{\prime}$. It contains $(n-1)$ diagonal blocks, each of the form

$$
N^{\prime}=\frac{-1}{A_{1}+A_{3}}\left(\begin{array}{cccc}
l_{j_{1}} & l_{j_{1}} & \ldots & l_{j_{1}} \\
\vdots & \vdots & \ddots & \vdots \\
l_{j_{s}} & l_{j_{s}} & \ldots & l_{j_{s}}
\end{array}\right)
$$

where $K_{2}=\left\{j_{1}, \ldots, j_{s}\right\}$. The determinant of the matrix in 9.8 is the same as the determinant of $I-N N^{\prime}$ where $N N^{\prime}$ is a square matrix consisting of $(n-1)$ diagonal blocks, each of the form

$$
N N^{\prime}=\frac{A_{2}}{\left(A_{2}+A_{3}\right)\left(A_{1}+A_{3}\right)}\left(\begin{array}{cccc}
l_{i_{1}} & l_{i_{1}} & \ldots & l_{i_{1}} \\
\vdots & \vdots & \ddots & \vdots \\
l_{i_{r}} & l_{i_{r}} & \ldots & l_{i_{r}}
\end{array}\right) .
$$

Since each diagonal block in $N N^{\prime}$ has rank 1 we directly calculate that the determinant is

$$
\left(1-\frac{A_{1} A_{2}}{\left(A_{1}+A_{3}\right)\left(A_{2}+A_{3}\right)}\right)^{n-1},
$$

but this is

$$
\left(\frac{A_{3}\left(A_{1}+A_{2}+A_{3}\right)}{\left(A_{1}+A_{3}\right)\left(A_{2}+A_{3}\right)}\right)^{n-1}
$$

and is always non-zero.

Corollary 9.9. Given any two tori $T^{I}$ and $T^{I^{\prime}}$ in $e^{-1}(S)$, if $T^{I \cap I^{\prime}}$ is the intersection torus, then locally the union $M\left(T^{I}\right) \cup M\left(T^{I^{\prime}}\right)$ is obtained via a plumbing construction where an appropriate collection of normal coordinates to $T^{I}$ over $T^{I \cap I^{\prime}}$ are identified with the usual coordinates in $T^{I^{\prime}}$.

Remark 9.10. When $T^{I}$ and $T^{I^{\prime}}$ have complementary dimensions then they intersect in the single point where all the links point in the same direction. Thus, their cap product will be 1 . 


\section{References}

[A] J.F. Adams, On the non-existence of elements of Hopf invariant one, Ann. Math., 72(1960), 20-104.

[C] J. F. Canny, The Complexity of Robot Motion Planning, Thesis, MIT, 1987.

[Ha] J.-C. Hausmann, Sur la topologie de bras articulès, Algebraic Topology Poznań 1989, 146-159, Lecture Notes in Math., 1474 Springer, Berlin, 1991

[HK] J.-C. Hausmann, A. Knutson, The cohomology ring of polygon spaces, Ann. Inst. Fourier (Grenoble), 48 (1998), 281-321.

[H] K.H. Hunt, Kinematic Geometry of Mechanisms, Oxford University Press, 1978.

[K1] Y. Kamiyama, Topology of equilateral polygon linkages, Topology and its Applications, 68(1996), 13-31.

[K2] Y. Kamiyama, Chern numbers of the moduli space of spatial polygons, Kodai Math. J., 23(2000), 380-390.

[K3] Y. Kamiyama, Euler characteristic of the moduli space of polygons in higher-dimensional Euclidean space, Kyushu J. of Math., 54(2000), 333-369.

[KTT] Y. Kamiyama, M. Tezuka, T. Toma, Homology of the configuration spaces of quasi-equilateral polygon linkages, Trans. AMS, 350(1998), 4869-4896.

[KT] Y. Kamiyama, M. Tezuka, Topology and geometry of equilateral polygon linkages in the Euclidean plane, Quart. J. Math., 50(1999), 463-470.

[KM1] M. Kapovich, J. Millson, On the moduli spaces of polygons in the Euclidean plane, Journal of Diff. Geometry, 42 (1995), 133-164.

[KM2] M. Kapovich, J. Millson, The symplectic geometry of polygons in Euclidean space, Journal of Diff. Geometry, 44 (1996), 479-513.

[KM3] M. Kapovich, J. Millson, Universality theorem for configuration spaces of planar linkages, Preprint, U. of Utah, (1998).

[KLOS] L.E. Kavraki and P. Švestka and J.-C. Latombe and M.H. Overmars, Probabilistic Roadmaps for Path Planning in High-Dimensional Configuration Spaces", IEEE Transactions on Robotics and Automation, 12, (1996), 566-580.

[SM] G. Shukla and A.K. Mallik, Detection of a crank in six-link planar mechanisms, Mechanism and Machine Theory, 35, (2000), 911-926. 
[ST] D.B.A Epstein, N.E. Steenrod, Cohomology Operations, Annals of Mathematics Studies \# 50, Princeton University Press.

[WXH] P. Watterberg, P.Xavier and Y. Hwang, Path Planning for Everyday Robotics, icra, (1997), 1170-1175.

This article may be accessed via WWW at http://www.rmi.acnet.ge/hha/ or by anonymous ftp at

ftp://ftp.rmi.acnet.ge/pub/hha/volumes/2004/n1a14/v6n1a14.(dvi,ps,pdf)

R. James Milgram milgram@math.stanford.edu

Department of Mathematics

Stanford University

Stanford California

J. C. Trinkle trink@cs.rpi.edu

Department of Computer Science

Rensselaer Polytechnic Institute

Troy, NY 12180-3590 